\title{
Spreading of bio-adhesive vesicles on DNA carpets $\dagger+$
}

\author{
Marie-Laure Hisette, ${ }^{a}$ Paula Haddad, ${ }^{b}$ Thomas Gisler, ${ }^{c}$ Carlos Manuel Marques ${ }^{a}$ and André Pierre Schröder ${ }^{* a}$
}

\author{
Received 9th October 2007, Accepted 16th January 2008 \\ First published as an Advance Article on the web 20th February 2008 \\ DOI: $10.1039 / \mathrm{b} 715530 \mathrm{a}$
}

Cell-adhesion events involve often the formation of a contact region between phospholipid membranes decorated with a variety of bio-macromolecular species. We mimic here such hairy bio-adhesive contact zones by spreading phospholipid vesicles onto surfaces carpeted with end-grafted $\lambda$-phage DNA. Our study reveals that the spreading front acts as a scraper that strongly stretches the DNA molecules, and that the multiple bonds created during vesicle spreading effectively staple the stretched chains in the gap between the membrane and the substrate. The scraping and stapling mechanisms revealed here for the long DNA molecules are expected to also play a role in actual bio-adhesion events of cell walls and tissues.

\section{Introduction}

Cell-adhesion events proceed by the formation of adhesive patches, the regions where the cell membrane and the adhering substrate are brought into intimate contact by ligand-receptor bonds. ${ }^{1,2}$ The formation of such adhesive zones has been explored in simple biomimetic systems by monitoring vesicles of phospholipid bilayers with known amounts of ligands, as they adhere on substrates with chosen densities of the corresponding receptors. ${ }^{3}$ However, little is known about the actual mechanisms and forces at play in the contact zone during the spreading process, particularly for membranes of practical interest that host not only ligands and receptors, but are also densely populated by many other bio-molecules. ${ }^{4}$ Bio-adhesion events are known to be determined by the mobility, strength, binding rate and lifetime of the discrete bonds that bring the two surfaces together. ${ }^{5,6}$ The interplay of these factors is better studied in biomimetic systems where the different parameters can be carefully tuned. ${ }^{7}$ We study the adhesion of giant unilamellar vesicles (GUVs) onto functionalized substrates with end-grafted DNA molecules. The vesicles are prepared by electroformation, ${ }^{8}$ from a mixture of two lipids (Avanti Polar Lipids): 1,2-dioleoyl-snglycero-3-phosphocholine (DOPC) and 1,2-distearoyl-sn-glycero3-phosphoethanolamine- $N$-(biotinyl(polyethylene glycol)2000) (DSPE-PEG2000-biotin) in different ratios. The vesicles interact with a glass substrate covered with streptavidin (see methods), a complementary receptor of the ligand biotin: the biotin-streptavidin pair forms a bond of more than $30 k_{\mathrm{B}} T$, one

aUniversité Louis Pasteur, Institut Charles Sadron, CNRS, 23 Rue du Loess, BP 84047, 67034 Strasbourg cedex 2, France; Fax: +33 388414 099; Tel: +33388414005

${ }^{b}$ Laboratório Nacional, Luz Sincrotron, Campinas, SP, BR-13084971, Brazil

'Fachbereich Physik, Universität Konstanz, Fach M621, Konstanz, 78457, Germany

$\dagger$ The HTML version of this article has been enhanced with colour images.

\$ Electronic supplementary information (ESI) available: A movie showing the spreading of a GUV over a DNA carpet. See DOI: $10.1039 / \mathrm{b} 715530 \mathrm{a}$ of the strongest bio-recognition affinities. ${ }^{9}$ The streptavidins on the substrate also anchor biotinilated $\lambda$-phage DNAs, with average end-grafting density of $c a$. 1 per $5 \mu \mathrm{m}^{2}$, well below the surface overlapping concentration. $\lambda$-DNA was stained with YOYO-1 (Molecular Probes), which is an intercalation fluorescent dye. ${ }^{10}$ We developed a new optical microscopy setup described in the method section, in order to observe simultaneously the adhesion of a giant vesicle on the glass substrate and the conformations of the grafted DNA molecules. The setup allows collecting images in two different observation modes. The first mode carries information from the interferences between the light reflected partially at the glass-water interface, and that reflected at the water-membrane interface. Such interference picture is similar to that obtained by reflection interference contrast microscopy (RICM), ${ }^{11}$ and provides information on the membrane substrate distance. The second acquisition mode provides a fluorescence image of the DNAs. The setup allows acquiring simultaneously images in the two modes at roughly 3 images per second.

\section{Results and discussion}

\section{The adhesion patch formation and the DNA stretching}

We show in Fig. 1 typical RICM and fluorescence images of one spread giant vesicle. The vesicle bears $2 \%$ of functionalized lipids corresponding to a ligand surface density $\sigma=1$ per $36 \mathrm{~nm}^{2}$. The adhesion patch is shown in Fig. 1a as a dark grey region, here with $22 \mu \mathrm{m}$ average diameter. Fig $1 \mathrm{~b}$ shows the corresponding fluorescence image. End-grafted DNA molecules outside the adhesive patch appear in the image as bright dots of roughly one-micrometre size. In the real time images of the movie available as ESI + , these dots display confined Brownian motion as expected from end-grafted polymers. ${ }^{12}$ DNA molecules within the patch are displayed as bright stripes oriented along the radial direction. These stripes end by a brighter dot-like region that we name the DNA "head", close to the patch periphery. In real time images, the stripes exhibit a vanishing mobility whereas the heads display confined Brownian motion similar to molecules outside the patch. One can also observe in the case of dense carpets a small number of collective spreading events where 

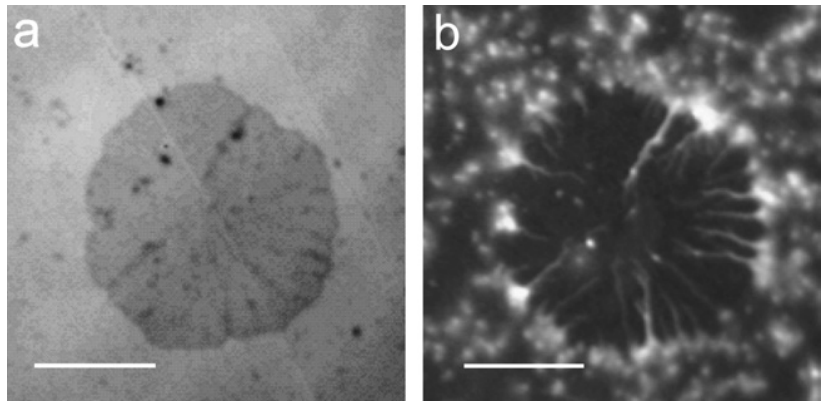

Fig. 1 Typical RICM and fluorescence images obtained from a biotinylated giant vesicle that has spread on a streptavidin-coated substrate carrying also end-grafted DNA molecules: (a) RICM image; the dark grey region corresponds to the membrane patch in close contact with the substrate; (b) fluorescence image of the same substrate zone, showing DNAs as bright structures. Scale bar represents $10 \mu \mathrm{m}$.

two DNA chains originally grafted nearby stretch as a single chain and split later on into two independent branches. The images in Fig. 1 show thus that spreading induces radial forces, strong enough to stretch the DNA chains. In order to reveal the intimate mechanisms that govern spreading we used our new optical setup for simultaneously acquiring RICM and fluorescence images.

The adhesion process is sketched in Fig. 2a, starting with the formation of the few first ligand-receptor bonds. The nucleated adhesion patch has a roughly circular shape, and it grows radially as the spreading front scraps the DNA molecules that it finds on its way. Spreading slows down exponentially as the patch radius approaches its final value. Fig. $2 b$ shows details of both the adhesion patch and the grafted DNAs at the same location and time during three different spreading stages. A complete movie of the spreading process can be found as ESIt. The upper half and lower half images display the same region of the sample, presented for simplicity in a mirror configuration. The upper half displays the fluorescence emitted by the DNAs and the lower part is the corresponding RICM picture. The first image on the left corresponds to the last frame acquired before nucleation. The interference rings of the RICM image show that the vesicle still has at this time a roughly spherical shape. The second image in the middle shows an intermediate spreading stage two seconds later. The adhesion patch is here shown as a homogenous grey disk in the lower half image. For DNAs with a grafting end under the adhesion patch, one can clearly distinguish in the upper half image a fluctuating coiled head at the border of the disk and a stretched stem connecting the head to the grafting point. The stretched part is strongly immobilized in less than one second, the time lag between three successive frames. Immobilization is certainly due to confinement by both the membrane and the biotin streptavidin bonds, in a stapling-like process. The last picture on the right shows the final state when the propagating front has stopped. The improved quality of the image has been obtained here by averaging over several tens of frames. The image clearly shows that most of the DNAs under the patch have been scraped during the adhesion process, a small number of chains remaining inside the patch in a coiled configuration. The scraping action of the propagating front is quantitatively analyzed in Fig. 2c. The left
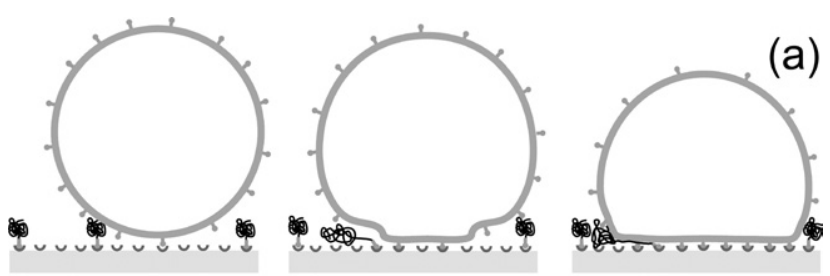

(b)
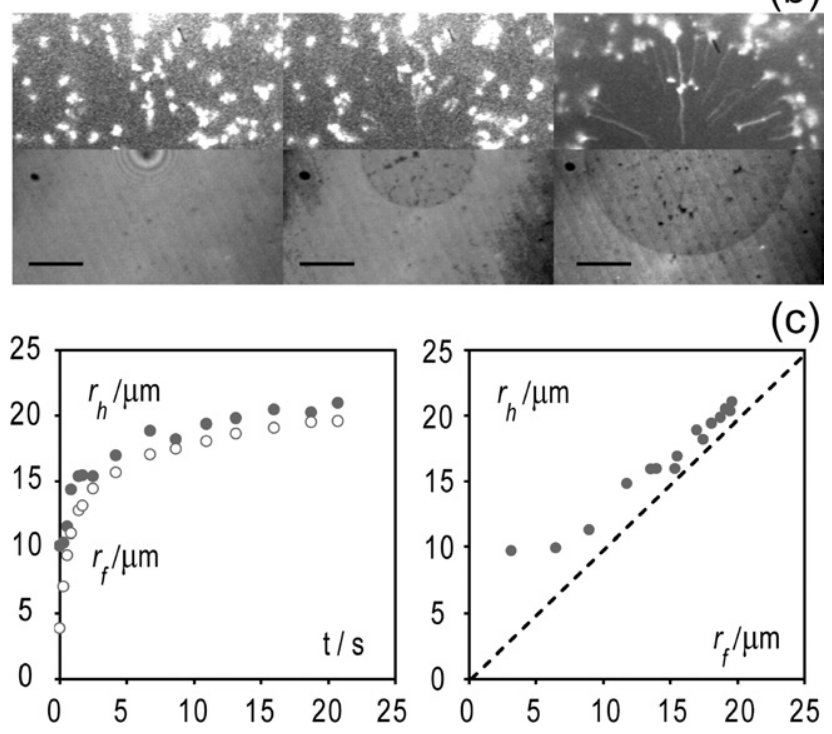

Fig. 2 Kinetics of adhesion patch formation. (a) schematic representation of the vesicle, substrate and DNA molecules at three different stages; (b) RICM and fluorescence images of the same surface region showing front propagation and the concomitant DNA stretching and immobilization; Scale bar represents $10 \mu \mathrm{m}$. (c) Comparison between the positions of the patch front $r_{\mathrm{f}}(\mathrm{O})$ and of one DNA free head $r_{\mathrm{h}}(\mathrm{O})$, showing that the vesicle spreading front scrapes the DNA carpet. Images were acquired at 3 images per second

plot shows the positions $r_{\mathrm{h}}$ of a DNA head $\S$ and $r_{\mathrm{f}}$ of the corresponding nearest point on the grey disk border, measured with respect to the geometrical centre of the disk. The $r_{\mathrm{f}}$ curve displays roughly exponential adhesion kinetics, typical of vesicle spreading $^{7}$ when diffusion of ligands does not play a significant role. The characteristic spreading time is here of the order of ten seconds. The right plot of Fig. 2c correlates $r_{\mathrm{h}}$ and $r_{\mathrm{f}}$ by plotting the position $r_{\mathrm{h}}$ of the head of the DNA as a function of $r_{\mathrm{f}}$ at successive times. It shows that the forces induced by the front propagation start to act on the DNA chain when the front comes within a few micrometres of the chain; from then on, the chain head keeps its position to the fore of the front until the propagation stops. The experiments show that a few moments before nucleation, the motion of the vesicle towards the surface induces a slight stretching of the DNA chains closer to the bottom of the vesicle. The maximum shear rate induced by a sphere of radius $R$ at a distance $h$ from the surface ${ }^{13}$ is written as $\dot{\gamma}=(-9 / 16)\left(6 R / h^{3}\right)^{1 / 2} \mathrm{~d} h / \mathrm{d} t$, giving under our conditions of approach $\dot{\gamma} \approx 2 \mathrm{~s}^{-1}$. The DNA stretching that we observe in

$\S$ The meaning of $r_{\mathrm{h}}$ is better seen in Fig. 4b, where the coil associated with the DNA head is clearly imaged. We measure $r_{\mathrm{h}}$ as the distance from the periphery of the coil to the center of the patch. 
our experiments is comparable to that reported in previous studies of tethered $\lambda$-DNA molecules in shear flow ${ }^{14}$ at the same shear rate. Once the nucleation has occurred, the adhesion front propagates with an initial velocity that depends on the ligand content and is here of the order of $10 \mu \mathrm{m} \mathrm{s}^{-1}$. Moreover, the absence of interference rings - see middle image in Fig. $2 b-$ in the RICM pattern shows that the front has a steep angle larger than $60^{\circ}$. Such steep angles are expected for fast membrane spreading. ${ }^{15}$ Also, the absence of any grey level variation near the adhesion patch edge shows that the rounding length $\xi=$ $(\kappa / \gamma)^{1 / 2}$, where $\kappa$ and $\gamma$ are respectively the bending modulus and the tension of the membrane, is smaller than our optical detection limit $\approx 0.2 \mu \mathrm{m}$. The nature of the hydrodynamic flow generated by the progression of the membrane wall mixes elongation and shear components. Since the membrane propagates with a steep angle, the flow in front of it has a symmetry similar to that of an incompressible fluid around an expanding cylinder: the velocity is radial $v(r)=r^{-1} r_{\mathrm{f}}(t) \mathrm{d} r_{\mathrm{f}}(t) / \mathrm{d} t$ and decreases with the distance $r$ from the centre of the patch. ${ }^{16}$ We can conclude from both graphs in Fig. 2c that the DNA starts to stretch from its undisturbed position at a point where the flow velocity reaches $c a .3$ micrometres per second, a value for which our DNA molecule is known to only stretch a few micrometres, ${ }^{17}$ consistent with the $r_{\mathrm{h}}-r_{\mathrm{f}}$ values shown in the figure. Close to the substrate the flow possesses a shear structure, with shear rate roughly given by $v(r) / h$ where $h$ is the thickness of the patch gap. For a maximum velocity of $10 \mu \mathrm{m} \mathrm{s}^{-1}$ and $h \sim 10 \mathrm{~nm}$, the shear rate takes a value $\gamma \approx 10^{3} \mathrm{~s}^{-1}$ and decreases exponentially to zero with the slowing down of the patch progression. Such high shear rates can strongly stretch a DNA molecule. ${ }^{14}$ However, in our geometry, the high shear region is confined to the immediate vicinity of the surface and of the propagation front. Thus, flow forces at play certainly help to orient those DNA segments closer to the front, but for the typical velocities of spreading in this study there are no long-range hydrodynamic forces able to stretch the DNA molecules more than three to four microns away from the front. At the end of the spreading process, the DNA molecules keep their final configurations for hours; partially confined and stapled by the biotin streptavidin bonds under the adhesion patch, and fully mobile for those segments outside the patch. The differences that remain at long time between $r_{\mathrm{h}}$ and $r_{\mathrm{f}}$ are likely due to several effects: steric interactions between the molecule and the membrane, membrane fluctuations, steep but finite spreading angle etc.

\section{Statistical analysis of the final configurations}

We study now the final configuration of the DNA carpet, from a statistical perspective. We have performed experiments with vesicles containing three different surface densities of ligands $\sigma_{1}=1$ per $3600 \mathrm{~nm}^{2}, \sigma_{2}=1$ per $360 \mathrm{~nm}^{2}$ and $\sigma_{3}=1$ per 36 $\mathrm{nm}^{2}$, and we display in Fig. 3 statistical results for the intermediate case $\sigma_{2}$. From all experiments, that concern tens of vesicles for each biotin content, we extracted the positions of the DNAs grafted sites (O), of the DNA heads $(O)$ and of the patch average radii $r_{\mathrm{p}}=r_{\mathrm{f}}(t \rightarrow \infty)$. The centre of the circle is then taken as the space origin and all dimensions are normalized with respect to $r_{\mathrm{p}}$, which allows plotting in a single diagram in Fig. 3a all the measured stretching events. The dominant

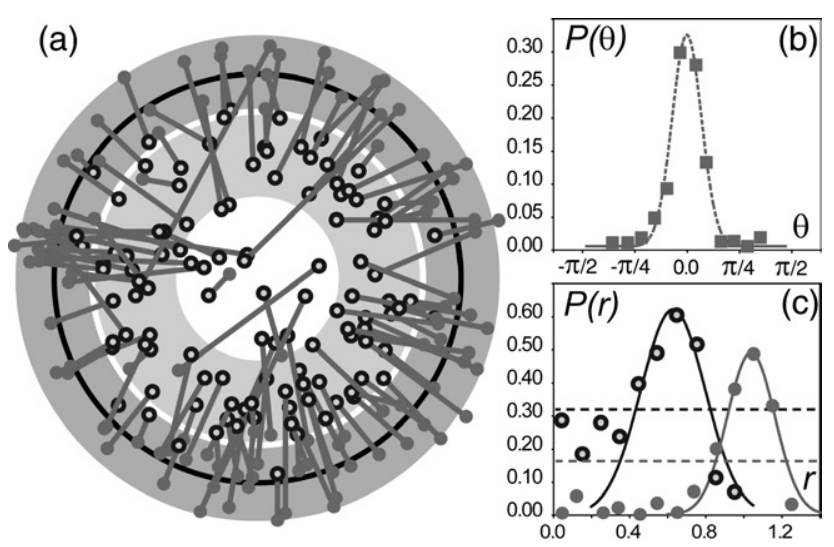

Fig. 3 Graphical representation of the distribution of stretched DNA under the adhesion patch. (a) The target-like picture displays the DNA-grafting sites (O), the DNA free ends or "heads" (O), and the end-to-end vector (-). The black circle corresponds to the adhesive patch border, and the shaded rings show above-average probability densities for the grafting sites (inner ring) and the chain heads (outer disk). Data was collected from several tens of vesicles. (b) Distribution $P(\theta)$ of the orientation $\theta$ of the end-to-end vector (-) with respect to the radial orientation of the adhesive patch. The Gaussian bell is a fit to the data with width $(\Delta \theta)=0.19$ radians. (c) Distribution $P(r)$ of the grafting sites (O) and free ends (O) with respect to a normalized patch radius $r\left(\int 2 \pi r P(r) d r=1\right)$. The dashed lines are average densities.

orientation is generated by the radial spreading forces. The distribution in Fig. $3 \mathrm{~b}$ shows the probability of finding a DNA oriented between $\theta$ and $\theta+\mathrm{d} \theta$ around the dominant $(\theta=0)$ radial orientation. The Gaussian shape is centred at $\theta=0$, the width of the distribution is $\left\langle\theta^{2}\right\rangle=0.19$. Fig $3 \mathrm{c}$ shows the radial probability density for finding grafted sites $(O)$ and chain heads (O) between radial positions $r$ and $r+d r$, where $r$ is the distance from the patch centre normalized by the patch radius $r_{\mathrm{p}}$. The head (O) radial density shows that a majority of the DNAs are scraped to the patch border. The Gaussian distribution is due both to the lack of circularity of the patch shape and to variations of the head position with respect to the patch border. A few DNAs escape full scraping, the whole molecule remaining confined within the gap. In most of such cases a frozen headlike part can still be observed. The most significant feature of the grafted sites (O) distribution is a low-density region in the centre of the patch. It indicates that GUVs tend to nucleate in the DNA carpet glades. We now summarize the statistical parameters of the distributions for the three biotin contents of this study. The Gaussian width $\left\langle\theta^{2}\right\rangle$ of the angle distribution assumes respectively the three values $0.19,0.19$ and 0.15 for the three densities $\sigma_{1}, \sigma_{2}$ and $\sigma_{3}$, a constant within errors. Equally, the width $(0.08,0.125$ and 0.15$)$ and the mean position $(1.03,1.04$ and 0.95$)$ of the renormalized head position distribution do not show a significant variation with the adhesion force.

\section{Investigation of the adhesion dynamics}

In order to investigate the interplay between the scraping front forces that stretch the DNA molecules and the stapling effects of the ligand-receptor pinning points, we analyse also the relative importance of the fluorescence intensity in the stretched 


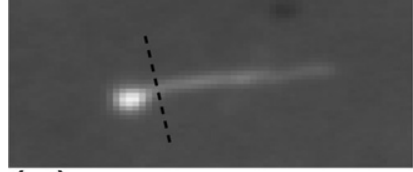

(a)

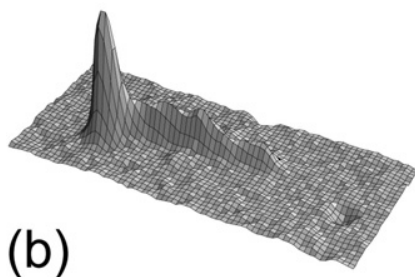

(b)

Fig. 4 Statistical information about the stretching imposed on the DNA molecules by the scraping and stapling process. (a) Original fluorescence image in grey levels. The DNA free end or "head" appears as a circular region of higher intensity. The length of the DNA tail is here $6.3 \mu \mathrm{m}$. (b) Three-dimensional representation of the same fluorescence intensity. Total and partial intensities described in the text are computed as the volumes under this surface. (c) Original (inset) and averaged values of the contour length density $n$ described in the text, as a function of the normalized radial position of the grafted chain end $r$. Values of $n$ close to one indicate complete stretching whereas larger $n$ values show looser configurations. Stretching increases with surface density of ligands $\sigma_{1}=1$ per $3600 \mathrm{~nm}^{2}(\boldsymbol{\square}), \sigma_{2}=1$ per $360 \mathrm{~nm}^{2}(\boldsymbol{\Delta})$ and $\sigma_{3}=1$ per $36 \mathrm{~nm}^{2}(\bullet)$.

part of the chain and in the head. Fig. 4a displays a typical fluorescence image of a stretched DNA molecule and Fig. 4b displays the corresponding three-dimensional representation of the intensity. The DNA segments confined within the adhesive patch display a low, roughly homogeneous intensity. The free head zone to the left of the dashed line in Fig. 4a shows a much larger intensity. By integration of the fluorescence intensities we get $i_{1}$, the integrated intensity in the head and $i_{2}$, the integrated intensity in the stalk. The relative intensity inside the patch is $i_{\mathrm{r}}=i_{2} /\left(i_{1}+i_{2}\right)$. By computing also the fraction $l_{\mathrm{r}}$ of the stretched DNA contour length in the patch, we can define the contour length density $n=i_{\mathrm{r}} / l_{\mathrm{r}}$. The value $n=1$ corresponds to a fully stretched chain. We plot in Fig. $4 \mathrm{c}$ the value of $n$ as a function of the relative grafting position $r=r_{\mathrm{g}} / r_{\mathrm{p}}$, where $r_{\mathrm{g}}$ is the distance of the grafting point to the centre of the patch, for the three different biotin surface densities in our study. Raw data appear noisy (inset in Fig. 4c) but averaged data (sliding average with window size $\Delta r=0.3$ ) show that in the range $r_{\mathrm{g}}$ $\in[0.15-0.85]$ two regimes are present: $n$ first grows linearly with $r_{\mathrm{g}}$, and then reaches a plateau, that depends on the biotin coverage. Thus, the biotin coverage $\sigma$ governs the DNA degree of folding in the patch gap via two mechanisms: the shear forces that act in the immediate vicinity of the advancing front and the bonding surface density below the patch are at play in the first regime, while the latter parameter seems to be the only one governing the second regime. We can further notice on Fig. $4 \mathrm{c}$ that the plateau value decreases with biotin coverage: $n \sim \sigma^{-1 / 4}$. These results will certainly be at the origin of future interesting theoretical work. From a practical point of view, we can see that within the range of adhesion strengths explored in this study, the degree of DNA stretching can be tuned by ligand content.

\section{Conclusion}

As a summary, our work uncovered the intimate mechanisms at play when a bio-adhesive membrane spreads on a substrate populated by large bio-macromolecules. When the bio-adhesive membrane spreads by the well-known rolling-out movement of the bilayer, as the many discrete ligand-receptor stickers progressively bind it to the substrate, we found that the advancing front of the adhesive region effectively generates outward forces on the bio-molecules anchored to the substrate, in a scraping movement able to fully stretch grafted DNA chains. Our results suggest that the scraping forces have a weak hydrodynamic component acting over a few microns and a strong steric component acting at membrane polymer contact. As the bilayer rolls over a stretched section of the DNAs it also staples it to the substrate, an inspiring collective mechanism that might remind us of Swift's Lilliputians stringing Gulliver to the ground. The discovery of scraping and stapling mechanisms in adhesive patch formation is likely to have a wide impact in bioadhesion: it will not only provide new guidelines for designing experiments and to devise new, more relevant biomimetic systems but it will also distinctly contribute to a better understanding of cell adhesion.

\section{Experimental}

\section{Streptavidin-coated substrates}

Glass coverslips (Labo Moderne, $24 \times 24 \mathrm{~mm}$ ) were first cleaned with Piranha $\left(50 \%\right.$ concentrated sulphuric acid $\mathrm{H}_{2} \mathrm{SO}_{4}$ and $50 \%$ hydrogen peroxide $\left.\mathrm{H}_{2} \mathrm{O}_{2}(30 \%)\right)$. The cleaned surfaces were amino-functionalized by immersion in a mixture of $98 \%$ ethanol and 2\% 3-aminopropyltriethoxysilane (Sigma-Aldrich). Followed a 30 minute exposure of the slide to a $8 \%$ solution droplet of glutaraldehyde (Polysciences) and subsequent rinsing with saline phosphate buffer (PBS). Streptavidin (Molecular Probes) was dissolved in PBS at $4 \mathrm{mg} \mathrm{ml}^{-1}$. A droplet of this solution was then deposited on the slide and rinsed after one hour with Tris-borate-EDTA buffer (TBE).

\section{Biotinylated vesicles}

Vesicles were prepared by the electroformation method from a mixture of two lipids (Avanti Polar Lipids): 1,2-dioleoyl$s n$-glycero-3-phosphocholine (DOPC) and 1,2-distearoyl$s n$-glycero-3-phosphoethanolamine- $N$-(biotinyl(polyethylene glycol)2000) (DSPE-PEG2000-biotin) in different ratios. The lipids were dissolved in a chloroform solution at a concentration of about $5 \mathrm{mg} \mathrm{ml}^{-1}$ and then spread onto one of the walls of the electroformation cell, made from glass slides with a metallic layer of indium tin oxide (ITO). After drying under vacuum, a 150 mOsm $\mathrm{kg}^{-1}$ sucrose solution was injected into the cell and an alternative electric field $(10 \mathrm{~Hz}, 1.5 \mathrm{~V})$ applied for one hour. Vesicles were then diluted in a $155 \mathrm{mOsm} \mathrm{kg}^{-1} \mathrm{TBE}$ solution and dropped onto the DNA carpet.

\section{DNA functionalization and grafting}

Double-stranded (Fermentas) $\lambda$-DNA (48502 base pairs) was end-functionalized with a molecule of biotin at one end and 
a molecule of DIG (digoxigenin) at the other end. This was obtained by using two single-stranded oligomers (MWGBiotech), one labelled with biotin and the other labelled with DIG. Each oligomer is complementary to one of the protruding ends of $\lambda$-DNA and can hybridize to the corresponding overhang. After reaction, end-labelled $\lambda$-DNA was separated from the remaining free oligomers with a Nick column (Amersham). The solution of $\lambda$-DNA was diluted by adding TBE to decrease the density of DNA onto the substrate. The resulting concentration of DNA was $c a .2 \mathrm{ng} \mu \mathrm{L}^{-1}$. $\lambda$-DNA was next stained with YOYO-1 (Molecular Probes), which is an intercalation fluorescent dye having a maximum adsorption at a wavelength of 491 $\mathrm{nm}$ and a maximum emission at $509 \mathrm{~nm}$. The dye-base pair ratio was $1: 5$, which increases the length of $\lambda$-DNA to $19.8 \mu \mathrm{m}(16.5 \mu \mathrm{m}$ without fluorescent dye). Variation of the substrate incubation time in DNA solutions from 30 minutes to 45 minutes allowed tuning the desired density of DNA. After incubation the substrate was rinsed with TBE in order to remove unbound DNA.

\section{Simultaneous RICM and fluorescence microscopy}

Simultaneous observation of vesicle adhesion by RICM and of DNA stretching by fluorescence was achieved using a homedesigned setup with a combination of filters and mirrors mounted on a Leica DMR microscope. The sample is illuminated in the reflection mode, using a mercury lamp (100 W), through a $100 \times$ oil immersion objective (Leica, NA 1.3). A band-pass filter (Omega Filters 455DF70) narrows the lamp spectrum to the $(425-490) \mathrm{nm}$ range. The filtered light beam is then partially reflected towards the sample by a dichroic mirror (Omega Filters 500DRLP); the mirror is fully transparent above $510 \mathrm{~nm}$, it reflects $100 \%$ of the light in the (440-480) nm range and roughly $50 \%$ in the (425-440) nm range. Light in the (425440) $\mathrm{nm}$ range travelling back from the sample after reflection is thus also transmitted through the same mirror with a transmission coefficient of roughly $50 \%$. It is this wavelength range that carries the reflection interference information. Light in the (440-480) nm range excites the fluorophores (YOYO-1) in our sample but is not transmitted $(0 \%)$ back through the dichroic mirror. Since this mirror is fully transparent above $510 \mathrm{~nm}$, it allows collecting the light emitted by the fluorophores. All the light transmitted through the dichroic mirror is separated later in the optical path by a 50/50 beamsplitter. The first beam crosses a bandpass filter (Omega Filters 535AF45, (510-560) nm) and is collected by a Hamamatsu C2400 video camera, providing the fluorescence image. The second split beam is collected by a Diagnostic Instruments NDIAG 1800 digital camera; it carries information from the interferences between the light reflected partially at the glass-water interface, and that reflected at the water-membrane interface. The latter observation mode is similar to the well known RICM mode, except that here, in order to avoid a too strong light attenuation, no analyzer and polarizer are used, and a larger bandwidth of light, i.e. (425-440) nm, is collected for the interference pattern formation. However, this does not modify the interferences pictures qualitatively. The NDIAG camera is synchronized by the Hamamatsu video signal. Simultaneous pictures are taken at approx. 3 images per second, and recorded on a PC.

\section{Acknowledgements}

We thank R. Lehner, J. Koota and I. Seuffert for experimental help with this project and Georg Maret for continuous support. This work was funded by the Deutsche Forschungsgemeinschaft.

\section{References}

1 G. I. Bell, Science, 1978, 200, 618.

2 P. Bongrand, Rep. Prog. Phys., 1999, 62, 921.

3 F. Brochard-Wyart and P. G. de Gennes, Proc. Natl. Acad. Sci. U. S. A., 2002, 99, 7854 .

4 M. Tanaka and E. Sackmann, Nature, 2005, 437, 656.

5 S. E. Chesla, P. Selvaraj and C. Zhu, Biophys. J., 1998, 75, 1553.

6 A. Pierres, A. M. Benoliel, C. Zhu and P. Bongrand, Biophys. J., $2001, \mathbf{8 1}, 25$.

7 D. Cuvelier and P. Nassoy, Phys. Rev. Lett., 2004, 93, 228101.

8 M. I. Angelova, S. Soléau, P. Méléard, J. F. Faucon and P. Bothorel, Prog. Colloid Polym. Sci., 1992, 89, 127.

9 P. C. Webe, D. H. Ohlendorf, J. J. Wendoloski and F. R. Salemme, Science, 1989, 243, 85.

10 H. S. Rye, S. Yue, D. E. Wemmer, M. A. Quesada, R. P. Haugland, R. A. Mathies and A. N. Glazer, Nucl. Acids Res., 1992, 20, 2803.

11 J. Rädler and E. Sackmann, J. Phys. II, 1993, 3, 727.

12 R. Lehner, J. Koota, G. Maret and T. Gisler, Phys. Rev. Lett., 2006, 96, 107801.

13 J. Happel and H. Brenner, Low Reynolds Number Hydrodynamics, Noordhoff International Publishing, Amsterdam, 1973.

14 P. S. Doyle, B. Ladoux and J. L. Viovy, Phys. Rev. Lett., 2000, 84, 4769.

15 S. R. Hodges and O. E. Jensen, J. Fluid Mech., 2002, 460, 381.

16 L. D. Landau and E. M. Lifshitz, Fluid Mechanics, Pergamon Press, Oxford, 1987.

17 T. T. Perkins, D. E. Smith, R. G. Larson and S. Chu, Science, 1995, 268, 83. 